\title{
Análise temporal da cobertura vegetal no Parque Estadual Cachoeira da Fumaça, ES, por meio de técnicas de sensoriamento remoto
}

\section{Temporal analysis of vegetation cover in the Waterfall Smoke State Park, ES, through remote sensing techniques}

Guilherme Silvério Aquino de Souza, Vinícius Nascimento Conrado, Bruno Ferraz Martins, Vagner Mauri Quinto e Elias Silva

\begin{abstract}
Resumo - O monitoramento ambiental de fragmentos florestais é um trabalho oneroso e demorado, por isso, o sensoriamento remoto (SR) por intermédio do índice de vegetação da diferença normalizada (NDVI) é uma ferramenta eficaz na identificação das áreas florestadas e que sofreram desmatamento. O satélite LANDSAT é uma importante fonte de base de dados para esse tipo de estudo, pois, possui imagens com mais de 29 anos de série temporal, o que possibilitou a criação dos mapas temáticos do Parque Estadual Cachoeira da Fumaça (PECF) localizado no Sul do Estado do Espírito Santo, e a identificação das áreas em que a vegetação arbórea sofreu desmatamento, não sofreu alteração e onde houve regeneração no parque. O PECF apresentou aproximadamente $72 \%$ da sua área sem sofrer alteração e $20 \%$ da sua área foi regenerada entre os anos de 1985 e 2010 , demonstrando eficiência do seu plano de manejo.
\end{abstract}

Palavras-chave: imagens orbitais, ndvi, monitoramento ambiental

\begin{abstract}
Environmental monitoring of forest fragments is a costly and time consuming job, so the remote sensing (RS) via the vegetation index (NDVI) is an effective tool to identify forested areas and suffered deforestation. The Landsat satellite is an important source of base data for this type of study because, have images with more than 29 years of time series, which enabled the creation of thematic maps of Waterfall Smoke State Park (PECF) located in the Southern State of Espírito Santo and the identification of areas where woody vegetation has suffered deforestation has not changed and where there was regeneration of the park. The PECF showed approximately $72 \%$ of its area as unchanged and $20 \%$ of its area was regenerated between the years 1985 and 2010, demonstrating the efficiency of its management plan.
\end{abstract}

Key words: orbital images, ndvi, environmental monitoring

\footnotetext{
*Autor para correspondência

Recebido para publicação em 20/12/2014; aprovado em 14/03/2015

*UFG - E-mail: guilhermesas@gmail.com
} 


\section{INTRODUÇÃO}

Desde a sua colonização, o Brasil se tornou em grande parte, um país agrícola, sendo que boa parte da área utilizada pela agricultura é resultante do desmatamento de biomas como a Mata Atlântica. Conforme Lima et al. (2005), o número de espécies em risco de extinção quase triplicou no Brasil em relação aos dois últimos levantamentos oficiais realizados.

O desmatamento descontrolado, seja para a exploração da madeira ou para a criação de novas fronteiras agrícolas ou pecuárias, obrigou o governo a regulamentar formas legais de preservação da biodiversidade dessas áreas vegetais.

As unidades de conservação (UC) foram criadas pela Lei Federal 9985/2000, tendo como objetivo a conservação, restauração e recuperação da biodiversidade das áreas protegidas (BRASIL, 2000).

O Sistema Nacional de Unidades de Conservação da Natureza (SNUC) divide as UC em de Proteção Integral (Estação Ecológica, Reserva Biológica, Parque Nacional, Monumento Natural e Refúgio da Vida Silvestre) e de Uso Sustentável (Área de Proteção Ambiental, Área de Relevante Interesse Ecológico, Floresta Nacional, Reserva Extrativista, Reserva de Fauna, Reserva de Desenvolvimento Sustentável e Reserva Particular do Patrimônio Natural)

A Cachoeira da Fumaça localizada entre os municípios de Alegre e Ibitirama no Sul do Estado do Espírito Santo, foi instituída como Reserva Florestal, ou seja, é uma UC. A reserva foi criada em 1984 com 24,2 ha e no ano de 1990 foi criado o Parque Estadual Cachoeira da Fumaça (PECF) (IEMA, 2009 citado por PRATER 2014). A área do parque no ano de 2009 foi ampliada para 162,5 ha (IEMA, 2014).

$\mathrm{O}$ uso do sensoriamento remoto (SR) por intermédio da produção de mapas temáticos como o índice de vegetação da diferença normalizada (NDVI) é uma ferramenta aplicável ao monitoramento da cobertura vegetal, possibilitando a identificação das áreas que foram restauradas e degradadas, caso haja. Segundo Moreira (2005), existe mais de 50 técnicas de análise e monitoramento temporal da vegetação, sendo o NDVI o mais utilizado.

Diante do exposto, este trabalho teve como objetivo avaliar as mudanças ocorridas na cobertura vegetal do PECF entre dois cenários: 1985 e 2010.

\section{MATERIAIS E MÉTODOS}

A área de estudo compreende o PECF, que se localiza nas divisas entre os municípios de Alegre e Ibitirama, no Sul do Estado do Espírito Santo (ES), nas coordenadas $N=7.716 .460$ $\mathrm{m}$ e E $=228.640 \mathrm{~m}$ (Datum SIRGAS 2000, Zona 24S, UTM), região conhecida como Caparaó Capixaba.

As imagens utilizadas foram adquiridas no catálogo de imagens do Instituto Nacional de Pesquisas Espaciais (INPE, 2014) sendo imagens do satélite LANDSAT 5 sensor TM (Mapeador Temático). As imagens eram datadas de 27/06/1985 e 31/05/2010, ou seja, ambas do mesmo período pós-chuva, mas de forma que a vegetação ainda expressava o seu potencial máximo devido a recarga do solo e do curso d'água. Foram escolhidos os anos de 1985 por ser o primeiro ano após a criação da Reserva e de 2010 por ser a imagem mais atual.

Conforme Liu (2006), as imagens de satélites são produtos brutos que devem ser corrigidas para que os seus pontos representem corretamente as coordenadas terrestres, uma vez que devido a sua resolução espacial e a curvatura da Terra, sofrem deslocamento das suas coordenadas. Segundo Novo (2010), as imagens de sensoriamento remoto apresentam mais fontes de distorções geométricas do que radiométrica.

As imagens foram processadas no software ArcGis 10®.

As correções radiométricas foram baseadas no Método de Uniformização das Variâncias (UMV), tendo as imagens do ano de 2010 como referência. Para os cálculos de ganho, offset e uniformização necessários para a normalização das imagens de 1985, foram realizados conforme as equações 1,2 e 3 .
Ganho $=\sqrt{\frac{\sigma_{R}^{2}}{\sigma_{A}^{2}}}$

Offset $=\mu_{\mathrm{R}}-\sqrt{\frac{\sigma_{\mathrm{R}}^{2}}{\sigma_{\mathrm{A}}^{2}}}{ }^{*} \mu_{\mathrm{A}}$

\section{$A^{\prime}=A *$ Ganho + Offset}

Em que:

$\sigma_{\mathrm{R}}^{2}$ : Variância da imagem de referência;

$\sigma_{\mathrm{A}}^{2}$ : Variância da imagem de ajuste;

$\mu_{\mathrm{R}}$ : Média da imagem de referência;

$\mu_{\mathrm{A}}$ : Média da imagem de ajuste;

$A^{\prime}$ : Imagem uniformizada;

$A$ : imagem de ajuste. 
As correções geométricas foram realizadas por meio do georreferenciamento das imagens com base no Sistema Integrado de Bases Geoespaciais do Estado do Espírito Santo (GEOBASES, 2014).

$\mathrm{NDVI}=\frac{\mathrm{TM} 4-\mathrm{TM} 3}{\mathrm{TM} 3+\mathrm{TM} 4}$

Em que:

NDVI: Índice de vegetação da diferença normalizada;

TM4: Banda 4 (infravermelho próximo);

TM3: banda 3 (vermelho)

Os mapas temáticos do NDVI gerados para os anos de 2010 e 1985 foram subtraídos, gerando uma nova imagem sintética denominada análise temporal da cobertura vegetal
O NDVI foi calculado conforme a equação 4, para os anos de 1985 e 2010 .

Tabela 1: Intervalo das classes de mudanças da cobertura vegetal, para o PECF, no Sul do Estado do Espírito Santo

\begin{tabular}{ccc}
\hline Classes & Intervalos \\
\hline Desmatamento & Mín., $\mu-\sigma$ & $-0,95 ;-0,11$ \\
Não Mudança & $\mu-\sigma, \mu+\sigma$ & $-0,11 ; 0,08$ \\
Regeneração & $\mu+\sigma$, Máx. & 0,$08 ; 0,33$ \\
\hline
\end{tabular}

Fonte: Adaptado de FERRARI et al. (2011)

que representa as mudanças ocorridas na cobertura vegetal conforme recomendação de MAAS (1999). Essa nova imagem foi reclassificada em 3 classes conforme a Tabela 1.

Após a reclassificação, a imagem foi convertida do formato raster para polígono e foi realizado o cálculo da área de cada classe de mudanças da cobertura vegetal.

\section{RESULTADOS E DISCUSSÃO}

O índice de vegetação da diferença normalizada para os anos de 1985 e 2010 podem se observados na Figura 1.

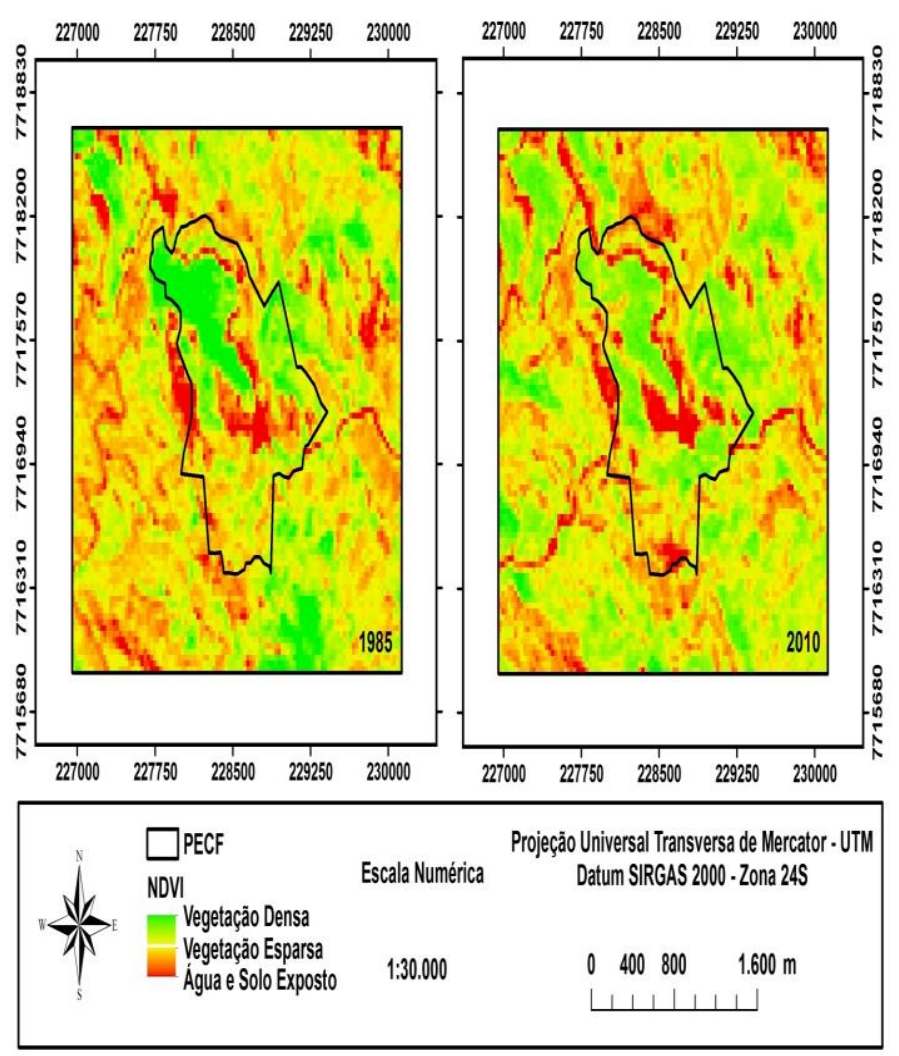

Figura 1: Índice de vegetação da diferença normalizada do PECF, Sul do Estado do Espírito Santo, nos anos de 1985 e 2010 Fonte: Os próprios autores 
Observando-se o NDVI dos anos de 1985 e 2010 (Figura 1) pode-se constatar a importância da criação das UC, pois, é dentro dos limites do PECF que se encontram as áreas de vegetação mais densa (coloração verde brilhante). Na Figura 1 no ano de 2010, ainda se pode observar o aumento da lâmina d'água em relação a 1985, evidenciando a necessidade de preservação da vegetação do entorno para recarga dos cursos d'águas.

O NDVI (Figura 1) permite ao parque elaborar estratégias de manejo, pois, identifica as áreas de solo exposto (coloração avermelhada) e com vegetação mais rala (esparsa) ou de pastagem (coloração verde clara).
Pela Figura 1, é possível se identificar a necessidade de uma intervenção antrópica na região Sul do PECF, pois, essa região encontra-se com solo exposto desde a sua criação até o ano de 2010, ou seja, o ambiente não conseguiu por si só estabelecer uma vegetação arbórea e densa nessa área. A região Sul do PECF sofreu processos de degradação entre os anos de 1985 e 2010, pois, apresentou coloração avermelhada no ano de 2010 (Figura 1), o que evidencia a presença de solo exposto.

As alterações ocorridas na cobertura vegetal do PECF entre os anos de 1985 e 2010 podem ser observadas na Figura 2.

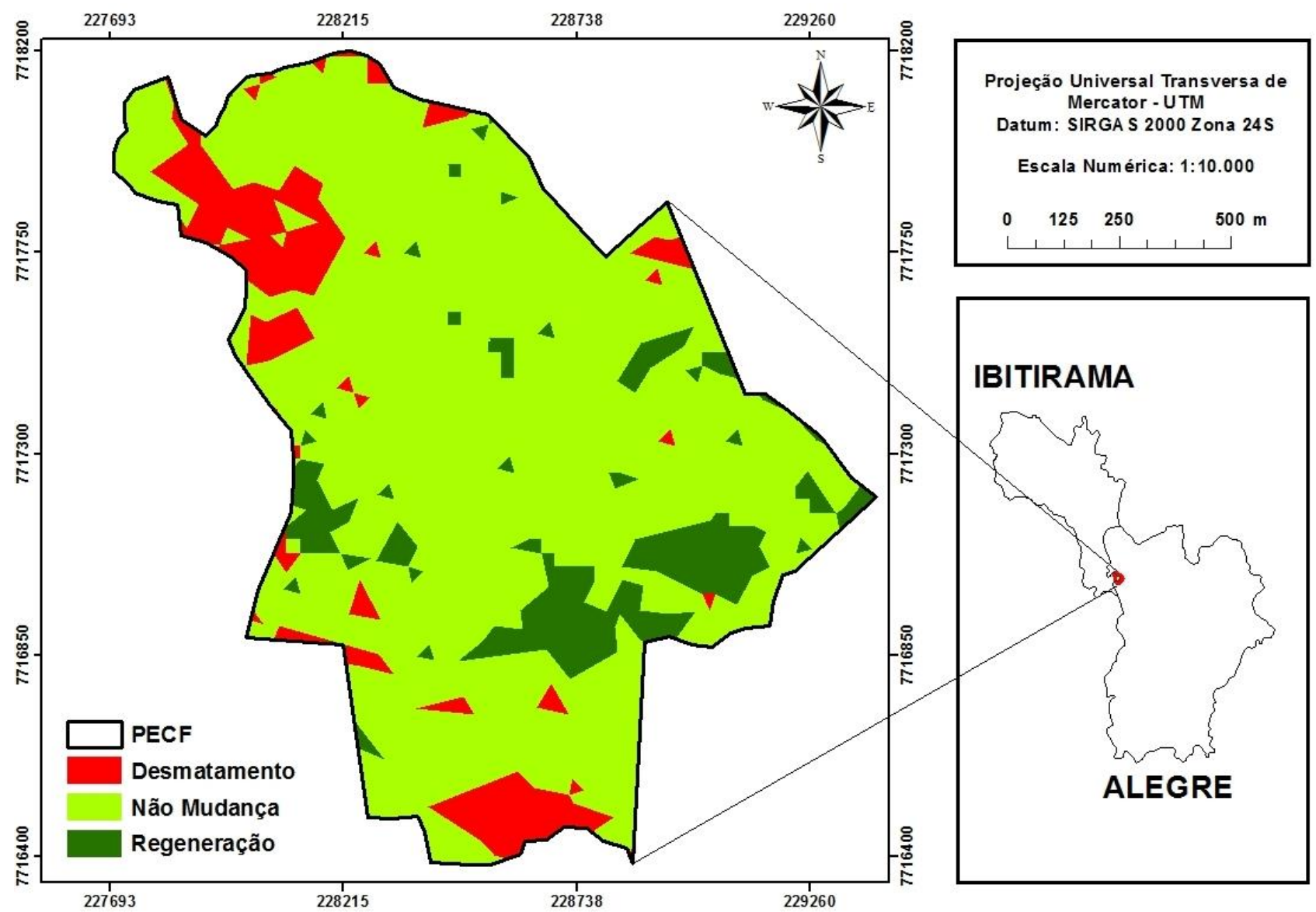

Figura 2: Análise temporal das mudanças da cobertura vegetal do PECF, Sul do Estado do Espírito Santo Fonte: Os próprios autores

O plano de manejo do PECF vem apresentando resultado satisfatório desde a criação do parque, tendo em vista que a maior parte do mesmo não sofreu alteração na sua cobertura vegetal conforme pode se observar na Figura 2.

Apesar da preservação da maior parte do parque, é possível se observar na Figura 2 que ainda existem áreas experimentando o processo de desmatamento, principalmente nas áreas de divisa. Esse processo pode ser devido a cultura dos proprietários vizinhos que ainda realizam atividades agrícolas ao entorno do parque, como o plantio de café e a criação de gado.

As áreas de desmatamento, não mudança e regeneração e suas respectivas porcentagens podem ser observadas na Tabela 2.

Tabela 2. Área das classes de alteração da cobertura vegetal

\begin{tabular}{ccc}
\hline Classes & Área (ha) & $\%$ área total \\
\hline Desmatamento & 14,16 & 8,71 \\
Não Mudança & 115,66 & 71,18 \\
Regeneração & 32,68 & 20,11 \\
\hline Total & 162,50 & 100,00 \\
\hline
\end{tabular}

Fonte: Os próprios autores 
Os 115,66 ha de não mudança representam $71,18 \%$ da área total do parque (Tabela 2), ilustrando a preservação da biomassa vegetal dentro do PECF desde a sua criação. Ainda analisando a Tabela 2 , pode se observar que 32,68 ha $(22,11 \%)$ foram regenerados e/ou recuperados por intermédio da ação antrópica, resultado este superior ao obtido por Thuler et al. (2012), demonstrando que entre os anos de 1985 e 1996 houve um significativo processo de regeneração. Estas áreas proporcionam a redução de processos erosivos, do assoreamento de cursos d'água e o aumento da área disponível para a fauna local.

Apenas 14,16 ha $(8,71 \%)$ do parque (Tabela 2) tiveram processos de desmatamento desde a criação do mesmo, resultado este semelhante ao obtido por Thuler et al. (2012), mas estes valores deveriam ser nulos. Desta forma, é premente a necessidade do planejamento das áreas de amortecimento ao redor do parque para que o mesmo não venha sofrer processos de degradação.

\section{CONCLUSÕES}

O PECF possui uma demanda da intervenção antrópica na sua região Sul para recomposição e estabelecimento de uma vegetação arbórea.

O plano de manejo do PECF apresentou resultados satisfatórios para a manutenção da preservação da vegetação.

Existe a necessidade da criação de um plano de manejo da zona de amortecimento ao redor do parque para contenção do desmatamento que ocorreu nas áreas de vizinhança.

O sensoriamento remoto por meio do uso de imagens orbitais e produtos como o NDVI é uma importante ferramenta para o monitoramento ambiental.

\section{AGRADECIMENTOS}

Os autores agradecem à CAPES (Coordenação de Aperfeiçoamento de Pessoal de Nível Superior), ao CNPq (Conselho Nacional de Desenvolvimento Científico e Tecnológico) e à FAPEMIG (Fundação de Amparo à Pesquisa do Estado de Minas Gerais) pela concessão de bolsas de estudo.

\section{REFERÊNCIAS BIBLIOGRÁFICAS}

BRASIL. Lei Federal n. 9.985, de 18 de julho de 2000. Institui o Sistema Nacional de Unidades de Conservação da Natureza (SNUC) e estabelece critérios e normas para a criação, a implantação e a gestão das unidades de conservação. Diário Oficial da República Federativa do Brasil, Brasília, jul. 2000.

FERRARI, J. L.; SANTOS, A. R.; GARCIA, R. F. Análise da vegetação por meio da subtração de imagem NDVI na sub-bacia hidrográfica do córrego do Horizonte, Alegre, ES. Engenharia Ambiental, v. 8, n. 3, p. 3-18. 2011.

GEOBASES - Sistema Integrado de Bases Geoespaciais do Estado do Espírito Santo. 2008. Disponível em: <http://www.geobases.es.gov.br/publico/Acesso Navegador.aspx id=142\&nome=NAVEGADOR_GEO BASES>. Acesso em: 5 abr. 2014.
IEMA (Instituto Estadual de Meio Ambiente e Recursos Hídricos). Parque Estadual Cachoeira da Fumaça. Disponível em: <http://www.meioambiente.es.gov.br/default.asp>. Acesso em: 5 abr. 2014.

INPE (Instituto Nacional de Pesquisas Espaciais). Disponível em: <http://www.dgi.inpe.br/CDSR/>. Acesso em: 5 abr. 2014.

LIMA, G. S.; RIBEIRO, G. A.; GONÇALVES, W. Avaliação da efetividade de manejo das unidades de conservação de proteção integral em Minas Gerais. Revista Árvore, v. 29, n. 4, p. 647-653, 2005.

LIU, W. T. H. Aplicações de sensoriamento remoto. Campo Grande - MS: Ed. Uniderp. 2006. 908 p.

MAAS, J. F. Monitoring land-cover changes: a comparison of change detection techniques. International Journal of Remote Sensing, v. 20, n. 1, p. 139-152, 1999.

MOREIRA, M. A. Fundamentos do sensoriamento remoto e metodologias de aplicação. 2005. Ed. UFV. $3^{\text {a ed. }}$ atual. ampl.- M.G. -Brasil. 208 p.

NOVO, E. M. L. M. Sensoriamento remoto: princípios e aplicações. 4 ed. São Paulo: Bluches, 2010.

PROATER 2011-2013 (Programa de Assistência Técnica e Extensão Rural). Planejamento e programação de ações 2011. Disponível em: <http://www.incaper.es.gov.br/proater/municipios/Capar ao/Alegre.pdf $>$. Acesso em: 5 abr. 2014.

THULER, L. H. M.; QUINTO, V. M.; BERNARDES, P. M.; BELTRAME, R. A.; FONSECA, A. S. Estudo da dinâmica florestal no Parque Estadual Cachoeira da Fumaça por meio de subtração de imagens NDVI. Enciclopédia Biosfera, v. 8, n. 14, p. 1251-1262, 2012. 